\title{
The harmonic mean renormalises random diffusion across a spatial multigrid
}

\author{
A. J. Roberts ${ }^{1}$ \\ (Received 28 January 2010; revised 9 April 2010)
}

\begin{abstract}
Most methods for modelling dynamics posit just two time scales: a fast and a slow scale. But many applications, such as the diffusion in a random media considered here, possess a wide variety of space-time scales. Consider the microscale diffusion on a one dimensional lattice with arbitrary diffusion coefficients between adjacent lattice points. I develop a slow manifold approach to model the diffusion, with some rigorous support, on a lattice that is coarser by a factor of four: the coarser scale effective diffusion coefficients are the harmonic mean of fine scale coefficients. Then iterating the analytic mapping of random diffusion from the finer grid to the coarser grid generates a hierarchy of models on a spatial multigrid across a wide range of space-time scales, all with rigorous support. The one step harmonic mean renormalises to harmonic means for the effective diffusion coefficients across the entire hierarchy.
\end{abstract}

http://anziamj.austms.org.au/ojs/index.php/ANZIAMJ/article/view/2176 gives this article, (C) Austral. Mathematical Soc. 2010. Published April 10, 2010. ISSN 1446-8735. (Print two pages per sheet of paper.) 


\section{Contents}

1 Introduction

C84

2 Coarsen lattice diffusion by a scale factor of two

C87

3 The slow manifold homogenises random diffusion

C90

4 Conclusion

C93

References

C93

\section{Introduction}

Research in the past decade provides new views of issues involved in modelling dynamics in classical physics and engineering $[15,17$, e.g.]. We seek to better understand the relation between microscale and macroscale models, and how to transform from one level of description to another [20, 18, e.g.].

For example, a critical issue in material science is the effective large scale properties of a composite material with significant microscopic structure. A canonical problem is the effective large scale diffusion through a domain with microscopic variations in diffusion coefficient $[1,2,19$, e.g.]. Here we transform diffusion on a fine grid, with arbitrary fine grid variations in diffusivity, into diffusion onto a coarser grid. The new result is that, to leading order, the coarse grid diffusion is a harmonic mean of the local fine grid diffusion.

Most methods for modelling dynamics posit just two time scales: a fast and a slow scale $[7,8,13$, e.g.]. But many applications possess a wide variety of interesting space-time scales [3, 7, e.g.]. Recent research introduced a methodology, with support from centre manifold theory, for changing the resolved spatial grid scale by just a factor of two [18]. Here we develop the methodology to better preserve the self-adjoint nature of random diffusion. 


$$
\begin{aligned}
& \frac{\mathrm{d} \mathrm{U}_{j}}{\mathrm{dt}} \approx \frac{1}{16}\left[\overline{\mathcal{K}}_{j-1 / 2} \mathrm{U}_{j-1}-\left(\overline{\mathcal{K}}_{j-1 / 2}+\overline{\mathcal{K}}_{j+1 / 2}\right) \mathrm{U}_{j}+\overline{\mathcal{K}}_{j+1 / 2} \mathrm{U}_{j+1}\right]
\end{aligned}
$$

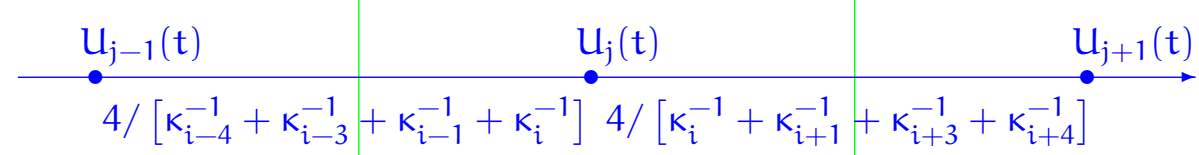

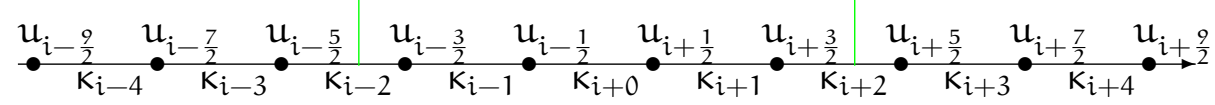

$$
\begin{aligned}
& \frac{d u_{i}}{d t}=\kappa_{i-1 / 2} u_{i-1}-\left(\kappa_{i-1 / 2}+\kappa_{i+1 / 2}\right) u_{i}+\kappa_{i+1 / 2} u_{i+1}
\end{aligned}
$$

FigurE 1: schematic transformation of diffusion on a fine grid (black) mapped, (1) $\mapsto(2)$, into equivalent diffusion on a grid coarser by a factor of four (blue) with diffusivities depending only on the local fine scale diffusivities.

As shown schematically in Figure 1, Section 2 invokes centre manifold theory to support that the self-adjoint evolution of discrete diffusion on a one dimensional, fine grid, with arbitrary spatially varying diffusivity,

$$
\frac{d u_{i}}{d t}=\kappa_{i-1 / 2} u_{i-1}-\left(\kappa_{i-1 / 2}+\kappa_{i+1 / 2}\right) u_{i}+\kappa_{i+1 / 2} u_{i+1}
$$

is mapped to self-adjoint dynamics on a coarser grid as

$$
\frac{\mathrm{d} \mathrm{U}_{j}}{\mathrm{dt}} \approx \frac{1}{16}\left[\overline{\mathcal{K}}_{j-1 / 2} \mathrm{U}_{j-1}-\left(\overline{\mathcal{K}}_{j-1 / 2}+\overline{\mathcal{K}}_{j+1 / 2}\right) \mathrm{U}_{j}+\overline{\mathcal{K}}_{j+1 / 2} \mathrm{U}_{j+1}\right]
$$

where the coarser scale diffusivities

$$
\overline{\mathcal{K}}_{j}=\frac{4}{\kappa_{4 j-2}^{-1}+\kappa_{4 j-1}^{-1}+\kappa_{4 j+1}^{-1}+\kappa_{4 j+2}^{-1}},
$$

are a harmonic mean over microgrid diffusivities and where the fine grid index $i=4 j$. This mapping of dynamics from a finer grid to a grid coarser 
via a factor of four, via finite elements formed from a small number of fine grid points, may then be iterated to generate a hierarchy of models across a wide range of spatial scales, with the theory of centre manifolds to support the modelling across the whole hierarchy of length scales.

It is well known that macroscale (system wide) homogenisation of microscale diffusivity variations (in 1D) is the harmonic mean [9, 13, e.g.]; here we find the new result that, because the harmonic mean of an harmonic mean is another harmonic mean, it is reasonable to homogenise diffusion with the harmonic mean at all scales. This approach promises to empower us with great flexibility in modelling other complex dynamics over multiple scales.

Most two scale modelling methods can also be applied over many scales. However, most established methods require each such application to be based upon a large 'spectral gap': a parameter such as $€$ measures the scale separation, and invoking "as $\epsilon \rightarrow 0$ " provides the extreme scale separation. In contrast, multigrid iteration for solving linear equations transforms between length scales that are different by (usually) a factor of two $[5,14,4$, e.g.]. Analogously, Section 3 introduces modelling dynamics on a hierarchy of length scales that differ by a factor of two and hence the 'spectral gap' is finite, not infinite as required by popular extant, non-multigrid, methods for modelling dynamics. The finite domain support provided by centre manifold theory empowers modelling dynamics with such a finite spectral gap.

The key to this modelling transformation of lattice dynamics is the coupling between finite elements. On the one hand we require coupling that ensures high order and classic consistency between the coarse model and the original fine lattice dynamics [12]; but on the other hand we require coupling which results in the coarse grid dynamics also preserving the self-adjoint symmetries of the fine grid lattice dynamics [16]. The innovation here is the coupling between finite elements that preserves the self-adjoint nature of diffusion as seen in the form of the coarse grid homogenisation (2).

Section 2 describes how to divide fine grid lattice dynamics into small finite elements (Figure 2), via interelement coupling rules that preserve self-adjoint 


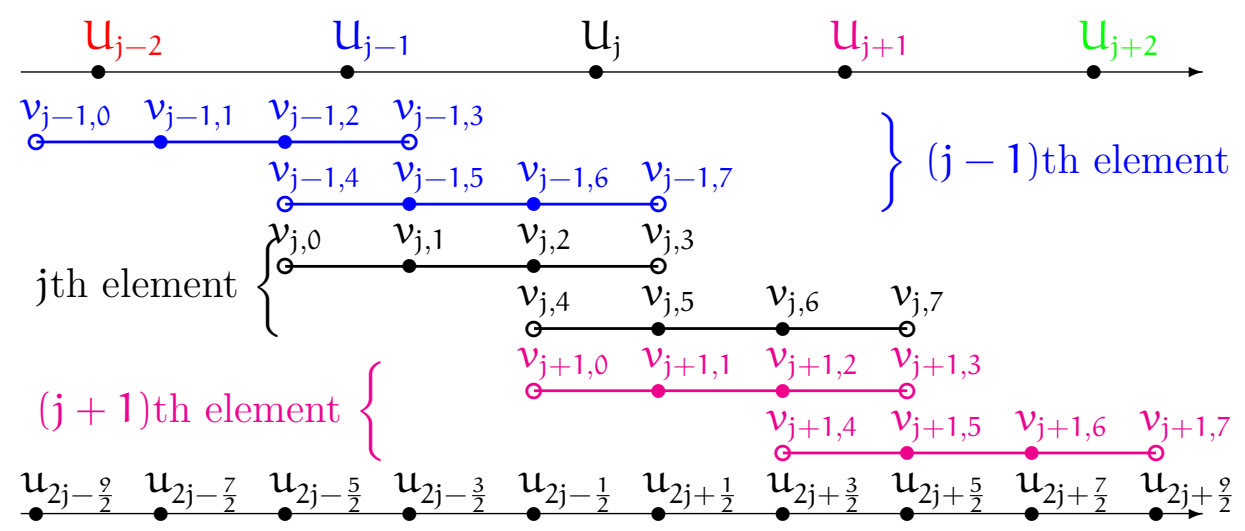

Figure 2: divide the fine lattice, bottom, into overlapping finite elements, middle, in order to derive evolution equations for coarse lattice variables $\mathbf{U}_{j}$, top. The fine lattice dynamics are applied to variables of the closed circles in the elements, whereas the variables of the open circles are used to implement self-adjoint coupling conditions.

symmetries, and then establishes the centre manifold support for the resulting coarse grid models. Although Section 2 seeks to coarsen the lattice by a factor of two, it eventuates that the leading order homogenisation coarsens by a factor of four as in $(1) \mapsto(2)$, and shown Figure 1.

\section{Coarsen lattice diffusion by a scale factor of two}

This section discusses an appealing way to transform the dynamics of variables $\mathfrak{u}_{i}(t)$ on a one dimensional fine lattice of spacing $h$, into dynamics of variables $U_{j}(t)$ on a coarser lattice of spacing $H=2 h$.

Figure 2 schematically shows that the $j$ th element stretches from a neighbourhood of $X_{j-1}$ to a neighbourhood of $X_{j+1}$. Figure 2 also shows each element 
is divided into two halves, and the variables duplicated so that, notionally, $u_{2 j}=v_{j-1,6}=v_{j, 2}=v_{j, 4}=v_{j+1,0}$ and $u_{2 j+1}=v_{j-1,7}=v_{j, 3}=v_{j, 5}=v_{j+1,1}$. We appear to need to split each element into two halves in order to preserve self-adjoint symmetry of the diffusion [16]. Embed the fine grid dynamics in these overlapping elements in a state space of double the dimensionality by treating $v_{j-1,6}$ and $v_{j, 2}$, and $v_{j, 5}$ and $v_{j+1,1}$ as independently evolving variables. For definiteness, suppose the original fine grid domain is $2 \mathrm{~m}$-periodic, so that the grid is divided into $\mathrm{m}$ elements that are $\mathrm{m}$-periodic in coarse grid index $j .{ }^{1}$ Analogous overlap of finite elements are increasingly being used in multiscale modelling $[8,19,10$, e.g.]. In essence we take one step back by the embedding, and then two steps forward via the centre manifold modelling.

The arbitrary diffusion, fine lattice, dynamics (1) embedded on the overlapping elements satisfy the differential-algebraic equation

$$
\mathrm{D} \dot{\overrightarrow{v_{j}}}=\mathrm{L}_{\mathrm{j}} \vec{v}_{\mathrm{j}}, \quad j=1, \ldots, \mathrm{m},
$$

where $\vec{v}_{j}=\left(v_{j, 0}, \ldots, v_{j, 7}\right), \mathrm{D}=\operatorname{diag}(0,1,1,0,0,1,1,0)$, and the diffusivity/ coupling matrix $L_{j}$ has four rows for fine scale dynamics and four rows for inter-element coupling parametrised by $\gamma$ and its complement $\gamma^{\prime}=1-\gamma$ :

$$
\begin{aligned}
\mathrm{L}_{j}= & {\left[\begin{array}{ll}
\mathrm{L}_{1,1} & \mathrm{~L}_{1,2} \\
\mathrm{~L}_{2,1} & \mathrm{~L}_{2,2}
\end{array}\right] \text { for the four sub-blocks } } \\
\mathrm{L}_{1,1}= & {\left[\begin{array}{cccc}
-\kappa_{2 j-2} & +\kappa_{2 j-2} & 0 & -\left(\gamma^{\prime}+\gamma \bar{\varepsilon}_{-}\right) \kappa_{2 j} \\
+\kappa_{2 j-2} & -\kappa_{2 j-2}-\kappa_{2 j-1} & +\kappa_{2 j-1} & 0 \\
0 & +\kappa_{2 j-1} & -\kappa_{2 j-1}-\kappa_{2 j} & +\kappa_{2 j} \\
-\kappa_{2 j}\left(\gamma^{\prime}+\gamma \bar{\varepsilon}_{+}\right) & 0 & +\kappa_{2 j} & -\kappa_{2 j}
\end{array}\right], } \\
\mathrm{L}_{2,2}= & {\left[\begin{array}{cccc}
-\kappa_{2 j} & +\kappa_{2 j} & 0 & -\kappa_{2 j}\left(\gamma^{\prime}+\gamma \bar{\varepsilon}_{-}\right) \\
+\kappa_{2 j} & -\kappa_{2 j}-\kappa_{2 j+1} & +\kappa_{2 j+1} & 0 \\
0 & +\kappa_{2 j+1} & -\kappa_{2 j+1}-\kappa_{2 j+2} & +\kappa_{2 j+2} \\
-\left(\gamma^{\prime}+\gamma \bar{\varepsilon}_{+}\right) \kappa_{2 j} & 0 & +\kappa_{2 j+2} & -\kappa_{2 j+2}
\end{array}\right], }
\end{aligned}
$$

$\mathrm{L}_{1,2}=\operatorname{diag}\left[\left(\gamma^{\prime}+\gamma \bar{\varepsilon}_{-}\right) \kappa_{2 j}, 0,0, \kappa_{2 j}\left(\gamma^{\prime}+\gamma \bar{\varepsilon}_{-}\right)\right]$,

${ }^{1}$ The resulting models, being local in space, are valid sufficiently far from physical boundaries. 


\section{$\mathrm{L}_{2,1}=\operatorname{diag}\left[\kappa_{2 j}\left(\gamma^{\prime}+\gamma \bar{\varepsilon}_{+}\right), 0,0,\left(\gamma^{\prime}+\gamma \bar{\varepsilon}_{+}\right) \kappa_{2 j}\right]$,}

where $\bar{\varepsilon}_{ \pm}$denotes the shift operators from one element to its neighbours: that is, $\bar{\varepsilon}_{ \pm} v_{j, i}=v_{j \pm 1, i}$. Then, since $\bar{\varepsilon}_{ \pm} \kappa_{2 j} v_{j, i}=\kappa_{2 j \pm 2} v_{j \pm 1, i}$, the system of matrix operators on the right-hand side of (3) is self-adjoint for all coupling parameter $\gamma$.

Centre manifold theorems support a coarse model Rigourous theoretical support is based upon the decoupled $(\gamma=0)$ dynamics. The embedded, fine grid dynamics (3) has an m-dimensional subspace $\mathbb{E}_{0}$ of equilibria: $\gamma=0$ and $v_{j, i}=\mathrm{U}_{\mathrm{j}}$ constant in each of the $\mathrm{m}$ elements. Here, consistent with Figure 2, choose the coarse variables $U_{j}$ to be the average of the mid-element values on the fine grid in each element. In the case when the diffusivities $\kappa_{i}$ are identical, the fine grid dynamics (3) on each element, linearised about $\mathbb{E}_{0}$, has spectrum proportional to $\{0,-2 / 3,-2,-4\}$ - the zero eigenvalue corresponds to the fine grid field $v_{j, i}$ being constant in each element. When $\kappa_{i}$ varies, elementary algebra shows that provided all $\kappa_{j}>0$ and all $2 \kappa_{2 j}\left(\kappa_{2 j-2}+\kappa_{2 j+2}\right)-\kappa_{2 j-2} \kappa_{2 j+2}>0$, then the spectrum remains as one zero eigenvalue with the other three being negative. Consequently, centre manifold theory assures us of the following corollary [6, 11, e.g.].

Corollary 1 (slow manifold) With the above proviso, in some finite neighbourhood of the subspace $\mathbb{E}_{0}$ :

1. there exists a $(\mathrm{m}+1)$ dimensional slow manifold $\mathcal{M}_{0}$ of the fine grid, element dynamics (3), and the slow manifold $\mathcal{M}_{0}$ may be written, for some $\vec{v}_{j}$ and $g_{j}, j=1, \ldots, m$, as

$$
\vec{v}_{j}=\vec{v}_{j}(\overrightarrow{\mathrm{U}}, \gamma) \quad \text { such that } \quad \dot{\mathrm{U}}_{\mathrm{j}}=\frac{\mathrm{du}_{\mathrm{j}}}{\mathrm{dt}}=\mathrm{g}_{\mathrm{j}}(\overrightarrow{\mathrm{U}}, \gamma) \text {; }
$$

2. the dynamics on $\mathcal{M}_{0}$ is emergent in that from all initial conditions in some finite neighbourhood of $\mathcal{M}_{0}$, there exists a solution of (5) approached exponentially quickly in time by the solution of (3); 
3. the order of error in the coupling parameter $\gamma$ of an approximation to the slow manifold $\mathcal{M}_{0}$ and its evolution, (5), is the same as the order of the residuals of the governing dynamics (3) when evaluated at the approximation.

I emphasise that this strong theoretical support applies in a finite neighbourhood of the decoupled case $\gamma=0$. I anticipate that the neighbourhood is large enough to include the physical case of full coupling, $\gamma=1$, as occurs in similar problems where evidence indicates both convergence and classic consistency at full coupling $[12$, e.g.].

\section{The slow manifold homogenises random diffusion}

Part 3 of the preceding corollary underlies the computer algebra that constructs the slow manifold model. In outline, given any approximation to the slow manifold and the evolution thereon, approximating (5), the algorithm evaluates the residual of the governing fine grid equations and coupling conditions (3), and then improves the approximation from the residual. This evaluation and improvement continues iteratively until the residual is small enough in terms of orders of the coupling parameter $\gamma$. The corollary then assures us that the slow manifold is approximated to errors of the same order in coupling parameter $\gamma$.

Executing the algorithm code $^{2}$ generates the following as the leading order

${ }^{2}$ http://anziamj . austms . org. au/ojs/index.php/ANZIAMJ/rt/suppFiles/2176 links to a supplementary file of the computer algebra code to construct the slow manifold. 
correction to the fine grid structure:

$$
\vec{v}_{j}=\left[\begin{array}{l}
1 \\
1 \\
1 \\
1 \\
1 \\
1 \\
1 \\
1
\end{array}\right] \mathrm{U}_{j}+\gamma \frac{1}{8} \mathcal{K}_{j}\left[\begin{array}{c}
-\kappa_{2 j}^{-1}-2 \kappa_{2 j-1}^{-1}-2 \kappa_{2 j-2}^{-1} \\
-\kappa_{2 j}^{-1}-2 \kappa_{2 j-1}^{-1} \\
-\kappa_{2 j}^{-1} \\
\kappa_{2 j}^{-1} \\
-\kappa_{2 j}^{-1} \\
\kappa_{2 j}^{-1} \\
\kappa_{2 j}^{-1}+2 \kappa_{2 j+1}^{-1} \\
\kappa_{2 j}^{-1}+2 \kappa_{2 j+1}^{-1}+2 \kappa_{2 j+2}^{-1}
\end{array}\right]\left(\mathrm{U}_{j+1}-\mathrm{U}_{j-1}\right)+\mathcal{O}\left(\gamma^{2}\right)
$$

where the local harmonic mean of the diffusivities is

$$
\mathcal{K}_{j}=\frac{4}{\kappa_{2 j-2}^{-1}+\kappa_{2 j-1}^{-1}+\kappa_{2 j+1}^{-1}+\kappa_{2 j+2}^{-1}} .
$$

In essence, this fine grid structure reduces to linear interpolation when all the diffusivities are the same, but reflects the internal variations in the fine grid field when the diffusivities vary. Terms of higher order in coupling $\gamma$ reflect more about the dynamics of fine grid structures, but are far to complicated to record here.

The computer algebra code correspondingly finds that the evolution on the slow manifold is

$$
\frac{d U_{j}}{d t}=\gamma^{2} \frac{1}{16}\left[\mathcal{K}_{j-1} \mathrm{U}_{j-2}-\left(\mathcal{K}_{j-1}+\mathcal{K}_{j+1}\right) \mathrm{U}_{j}+\mathcal{K}_{j+1} \mathrm{U}_{j+2}\right]+\mathcal{O}\left(\gamma^{3}\right) .
$$

Interestingly, there is no evolution at $\mathcal{O}(\gamma)$ in the coupling, we compute to $\mathcal{O}\left(\gamma^{2}\right)$ to find the leading order evolution. Consequently, our attempt to coarsen random diffusion by a factor of two in scale fails; our approach actually coarsens by a factor of four in scale - because the leading order model (8) only involves every second coarse grid point, $\mathrm{U}_{j}$ and $\mathrm{U}_{j \pm 2}$. Upon relabelling the coarse grid points, so that $\overline{\mathcal{K}}_{j}=\mathcal{K}_{2 j}$ for example, and evaluating at full coupling $\gamma=1$, the slow manifold evolution (8) becomes the homogenised coarse grid model (2) discussed in the Introduction. 
Observe that (8), and hence (2), are self-adjoint. But even more beautiful is that the effective diffusivity on the coarse grid is local: in (8) the diffusivity $\mathcal{K}_{j \pm 1}$ governing the flux between coarse grid points $X_{j}$ and $X_{j \pm 2}$ depends only upon the fine scale diffusivities between $X_{j}$ and $X_{j \pm 2}$, namely between $\kappa_{2 j}$ and $\kappa_{2 j \pm 4}$, from (7). This beautiful localisation is not built into our approach, but appears as a natural consequence of this scheme to preserve self-adjoint properties. ${ }^{3}$

Modelling should be transitive The slow coarse grid evolution (8) in turn may be modelled on an even coarser grid by the same process and same theoretical support to give a superslow model. Since a slow manifold is composed of subsets of solutions, and such a superslow manifold would be composed of a subset of the slow solutions, then the superslow manifold is composed of a subset of solutions of the fine scale dynamics. Our modelling is transitive: a coarser grid model of a coarse model will be the same as coarser grid model of the original fine grid dynamics.

Consequently, albeit approximate due to the truncation in coupling, the transformation $(1) \mapsto(2)$ of dynamics is justifiably applied across a multigrid hierarchy where each level of the grid is a factor of four coarser than its neighbouring finer grid. The transformation $(1) \mapsto(2)$ is effected by computing a harmonic mean of the finer grid diffusivities. But, as mentioned in the Introduction, a harmonic mea of a harmonic mean is just another harmonic mean, consequently at each level in the multigrid hierarchy the diffusivity at any locale at that level is a harmonic mean of the 'local' diffusivities at any of the levels below. This harmonic mean connects to the now classic 1D result from averaging $[9,13$, e.g.] that when the microstructure is on scale $\epsilon$ relative to the macroscale, then as $\epsilon \rightarrow 0$ ('infinite gap') the macroscale effective diffusion coefficient is the harmonic mean $\kappa_{H}=\left[\int_{D} \kappa(x)^{-1} d x\right]^{-1}$. The

${ }^{3}$ I also computed the model cubic in coupling $\gamma$. A sample of the 61,243 terms in its unfactored form suggest that these appealing properties also hold to at least the next order in coupling. 
novelty here is that centre manifold theory justifies the use of the harmonic mean of the fine scale diffusivities at all length scales between the microscale and the macroscale.

\section{Conclusion}

The finite domain support of centre manifold theory empowers great flexibility in modelling dynamics. Here we used the theory to justify modelling on a coarser grid the dynamics between interacting elements where each element possesses fine grid scale diffusion with arbitrary diffusion coefficients. The effective diffusivity on any coarser scale is then a harmonic mean of the fine scale diffusivities.

In applying the same technique to other problems, note that the transformation from one scale to another is generally nonlinear even for linear dynamics [18]; for example, here the harmonic mean is the nonlinear map for linear diffusion. Thus in other problems expect qualitatively different dynamics to emerge on different length scales through the nonlinear transformation of the model from one length scale to another.

Acknowledgement This research was supported by Australian Research Council grants DP0774311 and DP0988738. I thank Prof Yannis Kevrekidis and Dr Giovanni Samaey for stimulating discussions.

\section{References}

[1] Todd Arbogast and Kirsten J. Boyd. Subgrid upscaling and mixed multiscale finite elements. SIAM J. Numer. Anal., 44:1150-1171, 2006. doi:10.1137/050631811. C84 
[2] Achi Brandt. General highly accurate algebraic coarsening. Elect. Trans. Num. Anal., 10:1-20, 2000. http://www.emis.ams.org/ journals/ETNA/vol.10.2000/pp1-20.dir/pp1-20.html. C84

[3] Achi Brandt. Multiscale scientific computation: review 2001. In T. F. Chan T. J. Barth and R. Haimes, editors, Multiscale and Multiresolution Methods: Theory and Applications, pages 1-96. Springer-Verlag, Heidelberg, 2001. C84

[4] Achi Brandt. Methods of systematic upscaling. Technical report, Department of Computer Science and Applied Mathematics, The Weizmann Institute of Science, March 2006. http:

//wisdomarchive.wisdom.weizmann.ac.il:81/archive/00000398/. C86

[5] William L. Briggs, Van Emden Henson, and Steve F. McCormick. A multigrid tutorial, second edition. SIAM, 2nd edition, 2001. C86

[6] J. Carr. Applications of centre manifold theory, volume 35 of Applied Math. Sci. Springer-Verlag, 1981. C89

[7] J. Dolbow, M. A. Khaleel, and J. Mitchell. Multiscale mathematics initiative: A roadmap. Report from the 3rd DoE workshop on multiscale mathematics. Technical report, Department of Energy, USA, http://www.sc.doe.gov/ascr/mics/amr, December 2004. C84

[8] Weinan E, Bjorn Engquist, Xiantao Li, Weiqing Ren, and Eric Vanden-Eijnden. The heterogeneous multiscale method: A review. Technical report, http://www.math.princeton.edu/multiscale/review.pdf, 2004. C84, C88

[9] B. Engquist and P. E. Souganidis. Asymptotic and numerical homogenization. Acta Numerica, 17:147-190, 2008. C86, C92 
[10] Martin J. Gander and Andrew M. Stuart. Space-time continuous analysis of waveform relaxation for the heat equation. SIAM Journal on Scientific Computing, 19(6):2014-2031, 1998. C88

[11] Y. A. Kuznetsov. Elements of applied bifurcation theory, volume 112 of Applied Mathematical Sciences. Springer-Verlag, 1995. C89

[12] Tony MacKenzie and A. J. Roberts. Holistic discretisation ensures fidelity to dynamics in two spatial dimensions. Technical report, http://arxiv.org/abs/0904.0855v1, 2009. C86, C90

[13] G. A. Pavliotis and A. M. Stuart. Multiscale methods: averaging and homogenization, volume 53 of Texts in Applied Mathematics. Springer, 2008. C84, C86, C92

[14] A. J. Roberts. Simple and fast multigrid solution of Poisson's equation using diagonally oriented grids. ANZIAM J., 43(E):E1-E36, July 2001. http://anziamj . austms.org.au/ojs/index.php/ANZIAMJ/article/ view/465. C86

[15] A. J. Roberts. Low-dimensional modelling of dynamical systems applied to some dissipative fluid mechanics. In Rowena Ball and Nail Akhmediev, editors, Nonlinear dynamics from lasers to butterflies, volume 1 of Lecture Notes in Complex Systems, chapter 7, pages 257-313. World Scientific, 2003. C84

[16] A. J. Roberts. Choose interelement coupling to preserve self-adjoint dynamics in multiscale modelling and computation. Technical report, http://arxiv.org/abs/0811.0688, 2008. C86, C88

[17] A. J. Roberts. Normal form transforms separate slow and fast modes in stochastic dynamical systems. Physica A, 387:12-38, 2008. C84

[18] A. J. Roberts. Model dynamics across multiple length and time scales on a spatial multigrid. Multiscale Modeling and Simulation, 7(4):1525-1548, 2009. C84, C93 
[19] G. Samaey, I. G. Kevrekidis, and D. Roose. The gap-tooth scheme for homogenization problems. SIAM Multiscale Modeling and Simulation, 4:278-306, 2005. doi:10.1137/030602046. C84, C88

[20] G. Samaey, A. J. Roberts, and I. G. Kevrekidis. Equation-free computation: an overview of patch dynamics, chapter 8, pages 216-246. Oxford University Press, 2010. C84

\section{Author address}

1. A. J. Roberts, School of Mathematical Sciences, University of Adelaide, South Australia 5005, Australia.

mailto: anthony.roberts@adelaide.edu.au 